\title{
Cononsolvency Transition of Polymer Brushes: A Combined Experimental and Theoretical Study
}

\author{
Huaisong Yong ${ }^{1,2}$, Sebastian Rauch ${ }^{1}$, Klaus-Jochen Eichhorn ${ }^{1}$, Petra Uhlmann ${ }^{1}$ (D), \\ Andreas Fery ${ }^{1,2, *}$ and Jens-Uwe Sommer ${ }^{1,3, *}$ \\ 1 Leibniz-Institut für Polymerforschung Dresden e.V., 01069 Dresden, Germany; yong@ipfdd.de (H.Y.); \\ Rauch@ipfdd.de (S.R.); kjeich@ipfdd.de (K.-J.E.); uhlmannp@ipfdd.de (P.U.) \\ 2 Institute of Physical Chemistry of Polymeric Materials, Technische Universität Dresden, \\ 01062 Dresden, Germany \\ 3 Institute for Theoretical Physics, Technische Universität Dresden, 01062 Dresden, Germany \\ * Correspondence: fery@ipfdd.de (A.F.); sommer@ipfdd.de (J.-U.S.); \\ Tel.: +49-351-4658-225 (A.F.); +49-351-4658-750 (J.-U.S.)
}

Received: 30 April 2018; Accepted: 8 June 2018; Published: 11 June 2018

\begin{abstract}
In this study, the cononsolvency transition of poly( $N$-isopropylacrylamide) (PNiPAAm) brushes in aqueous ethanol mixtures was studied by using Vis-spectroscopic ellipsometry (SE) discussed in conjunction with the adsorption-attraction model. We proved that the cononsolvency transition of PNiPAAm brushes showed features of a volume phase transition, such as a sharp collapse, reaching a maximum decrease in thickness for a very narrow ethanol volume composition range of $15 \%$ to $17 \%$. These observations are in agreement with the recently published preferential adsorption model of the cononsolvency effect.
\end{abstract}

Keywords: cononsolvency; preferential adsorption; polymer brushes; PNiPAAm; ellipsometry

\section{Introduction}

Cononsolvency occurs if a mixture of two good solvents causes the collapse and segregation of polymer solutions into a polymer-rich phase in a certain range of compositions of these two solvents [1,2]. A prominent example [3] is poly ( $\mathrm{N}$-isopropylacrylamide) (PNiPAAm) in a water and methanol mixture. Although both solvents are good solvents for PNiPAAm, in a certain range of compositions of methanol and water PNiPAAm segregates from the mixed solution. Besides segregation in solutions, the cononsolvencyinduced volume phase transition of PNiPAAm gels has received much attention since the first paper published by T. Amiya et al. [4]. It is notable that a discontinuous, or jump-like, volume phase transition as it is observed for gels in such solvent mixtures would not be expected for a transition from a good to poor solvent within the common Flory-Huggins scheme because of the absence of translational degrees of freedom of the chains here [5,6]. This indicates a new kind of transition due to the solvent-cosolvent-polymer interaction. Given these observations, it is interesting to explore the possibility of a jump-like collapse transition for polymers on surfaces, in particular for polymer brushes.

Controlling the swelling of the polymer brushes and achieving a switching effect is quite important for the study of phase transitions [7-11] and applications of polymer brushes [12-14]; however, study of cononsolvency effects as a new switching effect in polymer brushes is still in its infancy stage. On the side of the experimental studies, previous studies mainly focused on how to use modern measurement techniques to monitor the cononsolvency transition of brushes. Liu and Zhang [15] used a quartz crystal microbalance with dissipation monitoring (QCM-D) to study the behavior of PNiPAAm brushes in water-methanol mixtures. They found a sharp cononsolvency transition, which they attributed to the formation of water-methanol complexes or clusters via hydrogen bonding. Later, 
S. Edmondson et al. [16] directly observed the volume change of polymer brushes by using ellipsometry to investigate the cononsolvency transition of poly(2-(methacryloyloxy)ethyl phosphorylcholine) brushes in alcohol-water mixtures. X. Sui et al. [17] observed morphological changes of PNiPAAm brushes by using surface probe microscopy in methanol-water mixtures. By using an extended surface forces apparatus, R. Espinosa-Marzal et al. [18] studied the impact of solvation on equilibrium conformation of dextran brushes in aqueous dimethyl sulfoxide mixtures. By combining the methods mentioned above, cononsolvency effects of polymer brushes have been related to applications for nanomaterials [19], tunable friction [20,21] and adhesive [22] properties of surfaces, as well as response of polymer brushes in liquid fluid media [23,24].

A possible molecular explanation for the new transition comes from atomistic simulations of single chains in the presence of a cosolution [25], and has been rationalized using the concept of preferential and non-specific adsorption of a cosolvent onto the polymer chain [26,27]. Based on the concept of preferential adsorption, one of the authors proposed a general model for a cononsolvency transition in polymer brushes [28], which has been extended recently to polymer solutions [29]. Chen et al. [30] also proposed a polymer lattice density functional theory (PLDFT) to investigate the cononsolvency phenomena related to polymer adsorption in a slit pore.

What is missing up to now is a physical understanding of the cononsolvency effect on real polymer brushes, which explains the equilibrium behavior and nature of the phase transition. The aim of this work is an investigation on the cononsolvency transition behavior in hydrophilic polymer brush systems in comparison with the theoretical predictions. We especially focus on a systematical VIS-spectroscopic ellipsometry (SE) measurement to study the properties of PNiPAAm brushes grafted on flat silicon surfaces in a mixture of ethanol and water.

\section{Materials and Methods}

Poly (glycidyl methacrylate) (PGMA, $M_{n}=10-20,000 \mathrm{~g} / \mathrm{mol}$ ) was purchased from Sigma-Aldrich (Darmstadt, Germany). Absolute ethanol (EtOH, 99.8\%) was acquired from Merck (Darmstadt, Germany). Tetrahydrofuran (THF, 99.98\%) and chloroform $\left(\mathrm{CHCl}_{3}, \geq 99 \%\right)$ were purchased from Acros Organics (Darmstadt, Germany). Purified water $\left(\mathrm{H}_{2} \mathrm{O}\right)$ was used from a Milli-Q Direct-8 system from Merck Millipore (Darmstadt, Germany). All chemicals were used as received, if not otherwise specifically noted. Highly polished single-crystal silicon wafers of $\{100\}$ orientation (Si-Mat Silicon Materials, Kaufering, Germany) were used as a substrate. End-functionalized PNiPAAm polymers $\left(M_{n}=50,200 \mathrm{~g} / \mathrm{mol}, M_{w} / M_{n}=1.28\right.$, monomer number of every chain is about 443$)$ with a terminal (tert-butyl protected) carboxy group were synthesized and characterized as described previously [31].

\subsection{Preperation of Polymer Brushes}

Silicon substrates $\left(13 \times 20 \mathrm{~mm}^{2}\right)$ were treated with EtOH in an ultrasonic bath for $10 \mathrm{~min}$, dried with a stream of nitrogen, and treated with oxygen plasma (440-G Plasma System, Technics Plasma GmbH, Wettenberg, Germany) for $1 \mathrm{~min}$ at $100 \mathrm{~W}$. Next a thin layer of PGMA (2.5 nm) was deposited by spin coating (Spin 150, SPS Coating, Ingolstadt, Germany) a PGMA solution in $\mathrm{CHCl}_{3}$ $(0.3 \mathrm{mg} / \mathrm{mL})$ with subsequent annealing at $110{ }^{\circ} \mathrm{C}$ in a vacuum oven for $20 \mathrm{~min}$ to react the silanol groups of the substrate with a fraction of the epoxy groups of PGMA, thus forming an anchoring layer equipped with the remaining epoxy groups for the following "grafting-to" process [32]. Afterwards, a filtered solution of end-functionalized PNiPAAm in THF $(9.0 \mathrm{mg} / \mathrm{mL})$ was spin coated onto the PGMA layer and subsequently annealed at $174{ }^{\circ} \mathrm{C}$ in a vacuum oven for $18 \mathrm{~h}$ (Scheme 1). To remove non-covalently bonded polymers, the resulting films were immersed first in $\mathrm{H}_{2} \mathrm{O}$, then extracted in $\mathrm{H}_{2} \mathrm{O}$ overnight, rinsed with EtOH, and dried in a stream of nitrogen.

Here we point out the primary advantage of the "grafting-to" method is that it is a technically simple processing step consuming low quantities of an accurately characterized pre-formed polymer. This means molecular weight, polydispersity, chain-end functionality, and especially the architecture can be designed very precisely, and thus comprehensive studies like the present one can be carried out. 

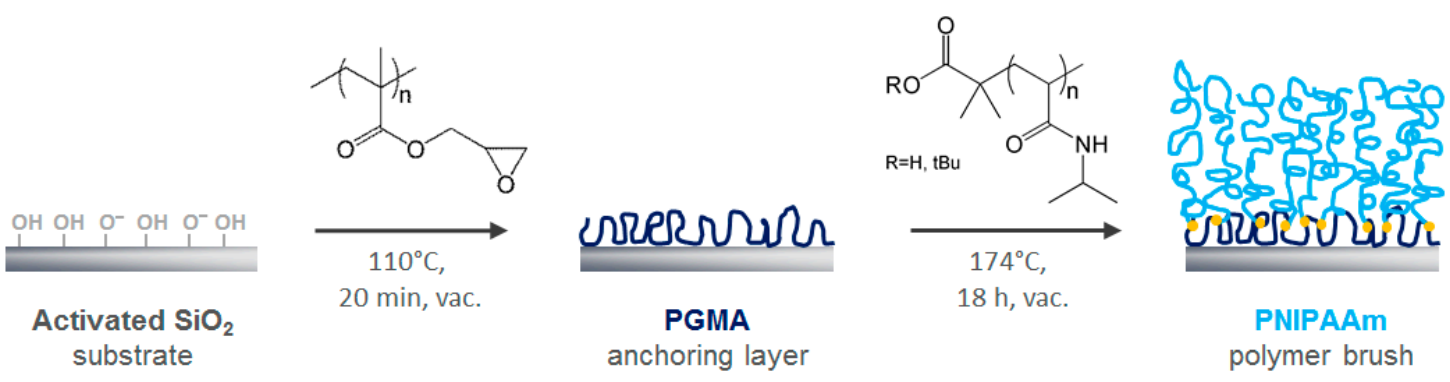

Scheme 1. Preparation of PNiPAAm brushes on silicon substrates using the "grafting-to" approach.

\subsection{VIS-Spectroscopic Ellipsometry Measurement}

A spectroscopic ellipsometer (alpha-SE, Woollam Co., Inc., Lincoln, NE, USA) equipped with a rotating compensator was used to measure the relative phase shift $(\Delta)$ and the relative amplitude ratio ( $\tan \Psi$ ) of the polymer brush films in the dry state, as well as in-situ in purified $\mathrm{H}_{2} \mathrm{O}$ and $\mathrm{H}_{2} \mathrm{O} / \mathrm{EtOH}$ mixtures within a batch cuvette (TSL Spectrosil, Hellma, Muellheim, Germany) at constant temperature of $25^{\circ} \mathrm{C}$ [33]. All solvent mixtures were prepared in the same day or one day before measurements and sealed in glass vials at room temperature. We always use the same brush by rinsing the solvent and introduce another solvent with help of a syringe-pump apparatus. The cuvette was flushed at least three times with the current solvent mixture right before measurement to avoid changes in the composition of the ambient. All measurements were performed between 400 and $800 \mathrm{~nm}$ at an angle of incidence $\Phi_{0}$ of $70^{\circ}$, which is close to the Brewster angle of silicon. To evaluate the index of refraction and thickness of the brush films in dry $(h)$ state and in situ $(H)$, a multilayer-box-model consisting of silicon, silicon dioxide, anchoring layer PGMA, and a polymer brush was assumed [31]. The refractive indices of the environment (water and $\mathrm{H}_{2} \mathrm{O} / \mathrm{EtOH}$ mixtures) were measured using a digital multiple wavelength refractometer (DSR-lambda, Schmidt + Haensch, Berlin, Germany). All data was acquired and analyzed using the Complete EASE $^{\circledR}$ software package (version 4.46). For the brush sample used in this work, we did three-time repeatable ellipsometry measurements. Result differences of these measurements were small, and as such, data reproducibility of our ellipsometry measurements were quite good.

From the determined film thickness $(h)$, polymer brush parameters like grafting density $(\sigma)$ and distance between anchoring points $(S)$ can be calculated by using Equation (1):

$$
\sigma=S^{-2}=N_{A} \rho h / M_{n}
$$

where $M_{n}$ is the number-average molecular weight of the polymer, $N_{A}$ is the Avogadro's number, $\rho$ is the polymer's melt density [34]. Here, $\rho$ is $1.1 \mathrm{~g} / \mathrm{cm}^{3}$ for PNiPAAm homo-polymer. For the interpretation of our measurements we have to assume a lateral homogeneous polymer profile. This is the case for the brush regime of strongly overlapping chains. To determine whether the grafted polymers are in the brush regime, the distance between grafting sites, $S$, should be small enough. For the case of a good solvent, $S$ should be much smaller than twice the radius of gyration of the chains $\left(R_{G}\right)$ :

$$
S / 2<R_{G}=\alpha N^{0.588},
$$

where $\alpha$ is a prefactor proportional to the monomer size and $N$ is the degree of polymerization; for the PNiPAAm polymer, $\alpha$ is estimated to be $0.3 \mathrm{~nm}[35,36]$. However, in the collapsed state, this condition is not sufficient. Here, so-called octopus-micelles can be formed if the grafting density is below the stretching threshold of $\sigma^{* *}$ [37],

$$
\sigma^{* *}=N_{A} \rho \alpha /\left(M_{0} \sqrt{N}\right),
$$


where $M_{0}$ is the molecular weight of repeat units of the polymer. If $\sigma>\sigma^{* *}$, the chains will form stretched brushes. This condition gives the upper estimate for the grafting density, which is necessary for a homogeneous brush. One might also use the overlap radius of gyration of the chains $\left(R_{P}\right)$ between collapsed single chains as a rough lower estimate for a homogeneous brush under the condition that chains collapse mostly, i.e., $R_{P}=\alpha N^{1 / 3}$ and $S / 2<R_{P}$, where the radius of gyration under poor solvent conditions is taken. We note that this estimate slightly underestimates the necessary grafting density. Basic physical parameters of PNiPAAm brushes used in this study are listed in the Table 1.

Table 1. Dry layer parameters (number-average molecular weight $M_{n}$, thickness $h$, grafting density $\sigma$, grafting distance $S$, and corresponding brush criteria) of polymer films, which were used in this study.

\begin{tabular}{ccccccccc}
\hline Polymer & $M_{n}(\mathrm{~g} / \mathrm{mol})$ & $\boldsymbol{h}(\mathbf{n m})$ & $\sigma\left(\mathrm{chains} / \mathbf{n m}^{2}\right)$ & $\sigma^{* *}\left(\mathrm{chains} / \mathbf{n m}^{2}\right)$ & $S(\mathrm{~nm})$ & $S /\left(2 R_{G}\right)$ & $S /\left(2 R_{P}\right)$ \\
\hline PGMA & $10-20,000$ & 2.5 & - & - & - & - \\
PNiPAAm & 50,200 & 14.9 & 0.20 & 0.084 & 2.3 & 0.1 & 0.5 \\
\hline
\end{tabular}

\section{Results and Discussion}

\subsection{Ellipsometry Analysis of the Cononsolvency Transition in PNiPAAm Brushes}

Although previous experimental studies [15-24] claimed that the cononsolvency transition of polymer brushes show features of a phase transition, so far, direct experimental evidence is missing. In order to check whether the cononsolvency transition of polymer brushes is an equilibrium transition, we measured swollen brush thickness as a function of time. Typical real-time measurements of $\mathrm{V}$ is-spectroscopic ellipsometry for swollen brush thickness of PNiPAAm are shown in Figure 1a-c. Except for the condition where the ethanol volume fraction is around $50 \%$, it is clearly seen that after about 5 min of continuous measurement, the brush system became stable. For the condition of the ethanol volume fraction around 50\%, in current measurement, the time needed to wait for the brush system to become stable was more than $20 \mathrm{~min}$. Thus, the data shown in Figure 1a-c supported the equilibrium nature of the cononsolvency transition that we report in this work.

Here, we point out that these differences in waiting time to reach equilibrium state may depend on the polymer characteristics itself and subsequent response. As the PNiPAAm polymer is quite thermally sensitive, even the enthalpy change via changing solvent composition can lead to changes in the polymer's configuration [38]. We also observed that with an increasing ethanol fraction, especially for the regime of high ethanol concentration, scatter in the real-time brush thickness increased. This might be explained due to lower optical contrast between the ambient ethanol and the thin PNiPAAm film. Note that all data about swollen brush thickness in the following are averaged values of real-time ellipsometry data in the stable regime, except when specifically indicated.

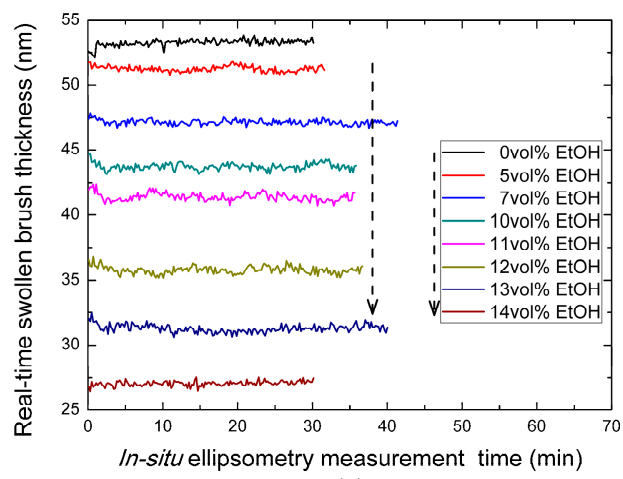

(a)

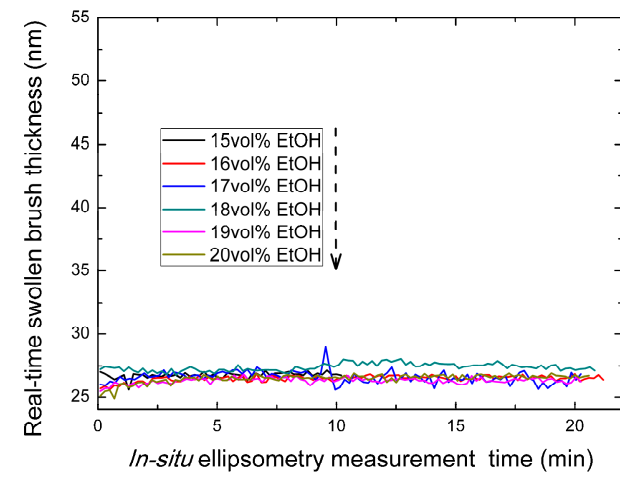

(b)

Figure 1. Cont. 


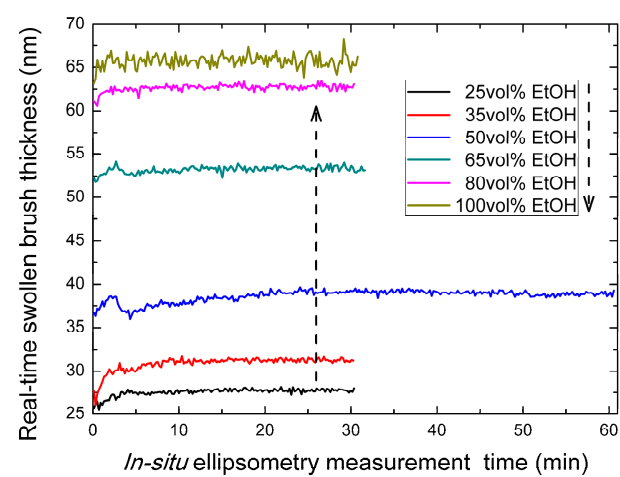

(c)

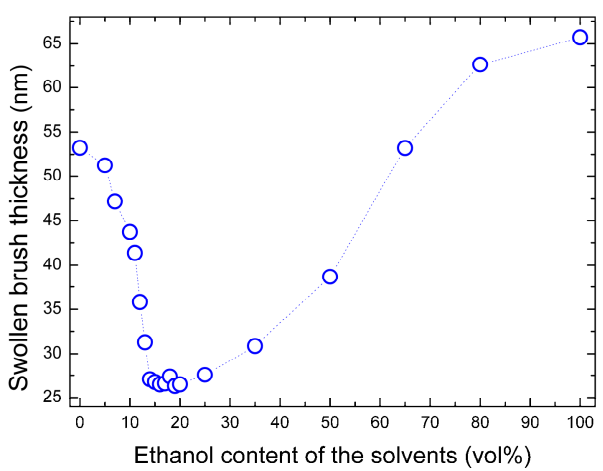

(d)

Figure 1. Swollen brush thickness of a PNiPAAm brush $\left(\sigma=0.20\right.$ chains $\left./ \mathrm{nm}^{2}\right)$ in aqueous ethanol mixtures as a function of (a-c) measurement time and (d) ethanol fraction obtained via Vis-spectroscopic ellipsometry. Note that the dashed line in Figure 1d is only used to guide eyesight. The error bars in Figure $1 \mathrm{~d}$ are neglected because they are quite small (around $0.2 \mathrm{~nm}$ ).

Figure $1 \mathrm{~d}$ summarises results for the PNiPAAm brush thickness (please see raw data in Appendix A.1) as a function of ethanol volume fraction $\left(\mathrm{V}_{\mathrm{E}}\right)$. We were able to qualitatively distinguish five solvent composition regimes: (i) the brush thickness of PNiPAAm was hardly affected by the change of $V_{E}$ when it was lower than 0.05; (ii) for the range of $0.05<V_{E}<0.15$, there was a sharp collapse transition of brushes, reaching a maximum decrease of thickness in a very narrow composition range of $0.15 \leq \mathrm{V}_{\mathrm{E}} \leq 0.17$ (Scheme 2); (iii) for the range of $0.17<\mathrm{V}_{\mathrm{E}}<0.3$, there was no obvious change of brush thickness in this regime; (iv) for the range of $0.3<\mathrm{V}_{\mathrm{E}}<0.8$, there was a re-entrant transition of brushes from a collapsed state to a swollen state; and (v) for the range of $V_{E}>0.8$, the brushes were fully re-swelling in ethanol-rich solvents. The brush thickness in pure ethanol $\left(V_{E}=1\right)$ was always thicker than in pure water $\left(\mathrm{V}_{\mathrm{E}}=0\right)$. This was a direct consequence of the stronger attraction between the alcohol and the monomer as compared to water and the monomer. This can be related with a larger excluded volume coefficient, and thus to stronger swelling. We also note that this phenomenon could be partially attributed to the molecular volume of ethanol being larger than the water's (Scheme 2, please see more details in the Appendix A.2).

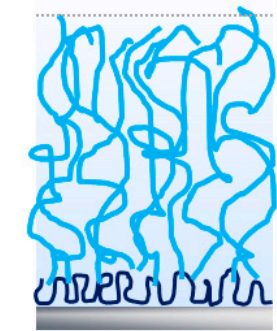

Stretched State pure $\mathrm{H}_{2} \mathrm{O}$

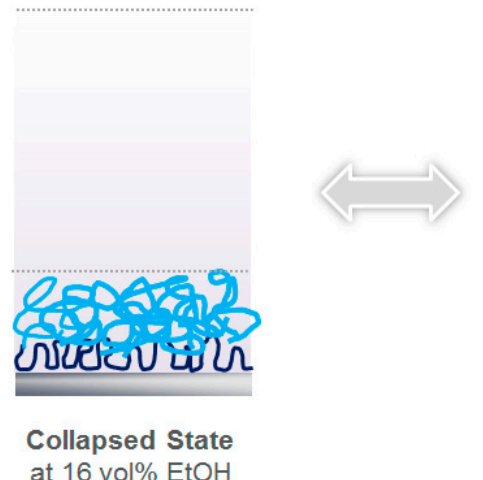

at $16 \mathrm{vol} \% \mathrm{EtOH}$

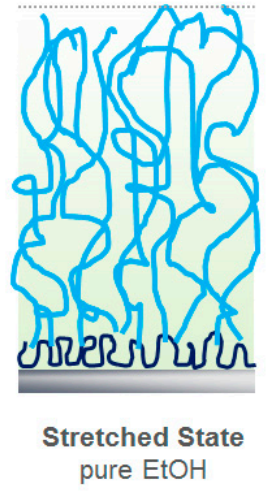

pure $\mathrm{EtOH}$

Scheme 2. Main cononsolvency transition regimes of a PNiPAAm brush in $\mathrm{H}_{2} \mathrm{O} / \mathrm{EtOH}$ mixtures.

In previous studies, Y. Yu et al. [21] also measured swollen thicknesses of PNiPAAm brushes with respect to a composition change of ethanol. Different from our results, they concluded that there was a non-sharp re-entrant transition of brushes by using ellipsometry measurements, reaching a maximum decreasing of thickness in a very broad composition range of $0.2<\mathrm{V}_{\mathrm{E}}<0.3$. The ellipsometry analysis of Reference [21] showed that the difference in swollen brush thickness in pure water and ethanol is negligible, which is in contrast with the corresponding AFM (Atomic force microscopy) measurement. 
Our own experience indicated that these discrepancies may be due to insufficient equilibration of the brush system in their ellipsometry measurements. Nevertheless, their AFM measurement results of the composition range [21] for the collapse transition with increasing ethanol concentration are consistent with our ellipsometry measurement results. We note that the grafting density in Reference [21] was chosen to be higher than in experiments reported here. According to the adsorption-attraction model, as discussed below, this should lead to an increase of the ratio between the height of the collapsed state and the height of the swollen state in pure water (deswelling ratio), which can indeed be concluded from the AFM measurement results of Reference [21].

Merely based on the results presented in Figure $1 \mathrm{~d}$, one should not conclude that the "swelling-to-collapse" transition with increasing ethanol concentration was sharper than the "collapse-to-swelling" transition with increasing ethanol concentration. Indeed, after transforming ethanol volume fraction $\left(\mathrm{V}_{\mathrm{E}}\right)$ into experimental chemical potential change, $\mu$ (please see more details in the Appendix A.3), it is clearly shown in Figure 2a that this "swelling-to-collapse-to-swelling" re-entrant transition curve became close to symmetric. According to the recently published adsorption-attraction model [28], the chemical potential change of solvent mixtures, not the volume fraction change of solvent mixtures, determines the equilibrium state of the brush. In particular, a nearly symmetric behavior of the brush thickness for collapse and reentry transitions is expected from the adsorption-attraction model [28].

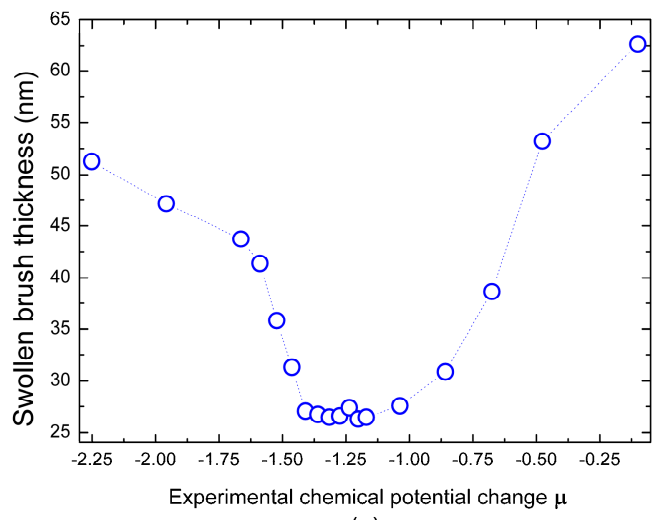

(a)

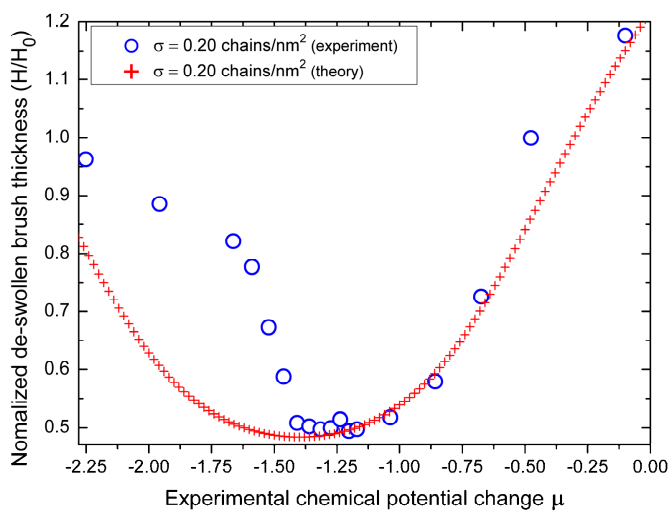

(b)

Figure 2. (a) Cononsolvency transition of a PNiPAAm brush $\left(\sigma=0.20\right.$ chains $\left./ \mathrm{nm}^{2}\right)$ in water/ethanol mixtures with reference to the swollen brush thickness as a function of experimental chemical potential change. Note that the dashed line is only used to guide eyesight. (b) Theoretical fitting of normalized de-swollen brush thickness plotted with respect to experimental chemical potential change in cononsolvency transition of a PNiPAAm brush.

\subsection{Application of the Adsorption-Attraction Model for Cononsolvency Transition in PNiPAAm Brushes}

To theoretically analyze the experimental results, we use the recently proposed adsorption-attraction model [28], which is based on the concept of preferential adsorption of a cosolvent onto the polymer as proposed by atomistic simulations [27]. The adsorption-attraction model predicts the free energy expression of the brush-solvent-cosolvent system using the equations listed below [28]:

$$
\begin{gathered}
f(\varphi, c)=f_{a d s}+f_{a t t r}+f_{b r u s h} \\
f_{a d s}=\varphi \ln \varphi+(1-\varphi) \ln (1-\varphi)-\mu \varphi-\varepsilon \varphi, \\
f_{a t t r}=-2 \varepsilon \gamma \varphi(1-\varphi) c \\
f_{b r u s h}=t \sigma^{2} /\left(2 c^{2}\right)+(1 / c-1-v \varphi) \ln (1-c-v \varphi c) .
\end{gathered}
$$


Here, $f(\varphi, c)$ is the free energy per monomer in the brush layer, where $c$ is the volume fraction of monomers in the brush layer. The first term, $f_{a d s}$, corresponds to the adsorption of an adsorbed cosolvent on the monomers of the chains. The number of cosolvent molecules per monomer is denoted by $\varphi$. The preferential adsorption is expressed by the energy gain $\varepsilon$ per adsorbed cosolvent. The first two terms in $f_{a d s}$ in Equation (4) correspond to the lattice gas state of a cosolvent on the polymer chains as a substrate. The chemical potential change of the solvent and cosolvent is denoted as $\mu$.

The term $f_{\text {attr }}$ denotes the mean-field attraction between monomers caused by forming a bridge due to the cosolvent. The strength of this additional attraction is given by $\gamma \varepsilon$, which takes into account bridging and size effects of the cosolvent by considering $\gamma \neq 1$. The last group of terms, $f_{\text {brush }}$, corresponds to the conformational free energy of the brush as a function of the volume fraction of monomers and of the grafting density, $\sigma$. The first term in $f_{\text {brush }}$ is the free energy for stretching, $t$ is a numerical prefactor, which accounts for the specific conformations of chains in a polymer brush diverging from Alexander-de Gennes approach; the second term is the Flory-Huggins free energy per monomer of the brush. Here, we took into account the effective increase of volume by adsorption of a cosolvent, where $v$ denotes the added volume fraction by full saturation of the polymer by a cosolvent. We note that no explicit interactions between the three components, other than the adsorption of a cosolvent on the chains, is considered. If taken pairwise, all components are fully miscible, and the collapse of the brush can only be the result of a cosolvent-induced attraction between the monomers.

The minimum for the free energy in Equation (4) can be obtained analytically for the "symmetric case" $v=0$. Here, one can show that the effect of the cosolvent can be mapped to an effective concentration-dependent $\chi$-parameter. As a consequence, the brush collapse can be of first order for a certain range of grafting densities and solvent selectivities. It is worth noting that the degradation of quality of a simple solvent cannot give rise to a step-like collapse transition because of the absence of translational entropy in this case. For the adsorption-attraction model, one can show that the effective attraction between monomers is also affected by the higher-order virial coefficients. Here, a coexistence between an effective 3-monomer attraction and an effective 2-monomer repulsion led to the first-order transition scenario. We note that experimental data indicated such as strong collapse behavior in the range of 15-20\% volume fraction of ethanol. For more details about the nature of the collapse/demixing transition due to preferential cosolvent adsorption we refer the reader to References [28,29].

The relation between the volume fraction of monomers in the brush layer and the height of the brush is $H=\sigma N / c$. The equilibrium height of the brush is given by the minimization of Equation (4) with respect to both free variables $c$ and $\varphi$. We display the numerical result together with the measurement data as a function of the chemical potential. Parameter values used in our theoretical fitting are $t=0.04, v=0.07, \gamma=1$, and $\varepsilon=1.25$. Note that in Figure $2 \mathrm{~b}, H_{0}$ is the swollen thickness of PNiPAAm brushes in pure water.

Based upon our theoretical fitting in Figure $2 b$, it was shown that the adsorption-attraction model can give a good description for a collapsed state of cononsolvency transition of polymer brushes. By way of qualitative analysis, it should be that the competition between attraction energy penalty of $f_{\text {attr }}$ and energy gains of $f_{\text {ads }}$ and $f_{\text {brush }}$ controls the "sharpness" of the cononsolvency transition; however, a closed analysis of the model with respect to the experimental results can only be done if essential parameters, such as grafting density or chain length, can be varied. Moreover, the exact value of the chemical potential difference in the mixed solvent is most important for a precise fitting since a shift of $\mu$ is related with a shift in the unknown solvent-solvent selectivity parameter $\varepsilon$, and thus changes the sharpness of the transition. We note that taking into account additional effects of the interaction between the components can lead to refinement of the prediction, in particular, the location and strength of the collapse transition. It has already been pointed out in previous studies that interaction between the solvent and cosolvent could play a role in the cononsolvency transitions of polymer brushes $[15,18]$. We also note that the effect of polydispersity is not taken into account in 
Equation (4). Without doubt, polydispersity plays an important role in affecting properties of polymer brushes [39-41].

\section{Conclusions}

In the present study, we investigated the cononsolvency effect of PNiPAAm brushes with moderate grafting density in aqueous ethanol mixtures. We have used Vis-spectroscopic ellipsometry measurements to prove the hypothesis that the cononsolvency transition of PNiPAAm brushes consists of a volume phase-like transition. For low ethanol fractions in the system, there was a sharp collapse of the brushes, reaching a minimum in film thickness in a very narrow ethanol volume fraction range from $15 \%$ to $17 \%$. This was followed by a reentry transition at a volume fraction in the range of $30 \%$ to $80 \%$. If the chemical potential is taken as the control variable, both transitions were qualitatively similar, while the reentry transition was weaker, i.e., not as jump-like as the collapse transition. These general features have recently been predicted by a mean-field type adsorption-attraction model, which can be a good starting point to qualitatively describe the cononsolvency transition of PNiPAAm brushes. Without a doubt, grafting density, molecular weight, and the molecular weight distribution had an important influence on the properties of polymer brushes. The theoretical model made clear predictions for the influence of the grafting density: Increasing the grafting density should have increased the deswelling ratio, but the grafting density only had a little effect on the solvent-composition location of the minimum of the brush height. In future, we will further study how these parameters affect the cononsolvency transition properties of polymer brushes. This will allow for a comparison of scaling predictions of the model, and for an analysis of the various molecular contributions to the cononsolvency effects. Furthermore, computer simulations can address more detailed properties, such as the density profile of monomers.

Author Contributions: H.Y. conducted the experiments and analyzed the data. S.R., A.F. and J.-U.S. directed this work. All authors wrote the paper and approved the final version of the manuscript.

Funding: This research was funded by the Deutsche Forschungs Gemeinschaft (DFG) under Grant No. SO-277/13.

Acknowledgments: The authors thank André Galuschko, Roland Schulze and Eva Bittrich for valuable discussions.

Conflicts of Interest: The authors declare no conflict of interest.

\section{Appendix A.}

Appendix A.1. Raw Data of Swollen Thickness of PNiPAAm Brushes

Table A1. Raw data of swollen brush thickness of a PNiPAAm brush plotted in Figure 1d.

\begin{tabular}{lccccccc}
\hline Ethanol Concentration & $0 \mathrm{vol} \%$ & $5 \mathrm{vol} \%$ & $7 \mathrm{vol} \%$ & $10 \mathrm{vol} \%$ & $11 \mathrm{vol} \%$ & $12 \mathrm{vol} \%$ & $13 \mathrm{vol} \%$ \\
\hline Brush Thickness $(\mathbf{n m})$ & 53.2 & 51.3 & 47.2 & 43.7 & 41.4 & 35.8 & 31.3 \\
\hline
\end{tabular}

Table A2. Raw data of swollen brush thickness of a PNiPAAm brush plotted in Figure 1d.

\begin{tabular}{lccccccc}
\hline Ethanol Concentration & $14 \mathrm{vol} \%$ & $15 \mathrm{vol} \%$ & $16 \mathrm{vol} \%$ & $17 \mathrm{vol} \%$ & $18 \mathrm{vol} \%$ & $19 \mathrm{vol} \%$ & $20 \mathrm{vol} \%$ \\
\hline Brush Thickness $(\mathbf{n m})$ & 27.1 & 26.8 & 26.5 & 26.6 & 27.4 & 26.3 & 26.5 \\
\hline
\end{tabular}

Table A3. Raw data of swollen brush thickness of a PNiPAAm brush plotted in Figure 1d.

\begin{tabular}{ccccccc}
\hline Ethanol Concentration & $25 \mathrm{vol} \%$ & $35 \mathrm{vol} \%$ & $50 \mathrm{vol} \%$ & $65 \mathrm{vol} \%$ & $80 \mathrm{vol} \%$ & $100 \mathrm{vol} \%$ \\
\hline Brush Thickness $(\mathbf{n m})$ & 27.6 & 30.9 & 38.6 & 53.2 & 62.6 & 65.7 \\
\hline
\end{tabular}

Appendix A.2. The Role of Volume of Solvent Molecules in the Swelling of PNiPAAm Brushes

Based on scaling theory [6], the brush's height, $H$, on a flat surface can be expressed as

$$
H=k b^{\frac{1}{x}} S^{1-\frac{1}{x}} N,
$$


where $b$ is the actual monomer size, $S$ is the average distance between two adjacent grafted polymer chains, $N$ is the degree of polymerization, $k$ is a numerical prefactor that depends on temperature and polymer's chemical structure, and $x$ is the Flory exponent.

The size of a water molecule is about $0.28 \mathrm{~nm}$, the size of an ethanol molecule is about $0.44 \mathrm{~nm}$, and the size of a PNiPAAm repeat unit is about $0.70 \mathrm{~nm}$ [42]. Here, we assume that in pure solvents, every repeat unit of PNiPAAm adsorbs a solvent molecule. Then, the actual monomer size of PNiPAAm in pure ethanol is about $1.14 \mathrm{~nm}(=0.70 \mathrm{~nm}+0.44 \mathrm{~nm})$, and the actual monomer size of PNiPAAm in pure water is about $0.98 \mathrm{~nm}(=0.70 \mathrm{~nm}+0.28 \mathrm{~nm})$. Then the ratio of brush height in pure ethanol $H_{E}$ to the brush height in pure water $H_{W}$, is $H_{E} / H_{W}=(1.14 / 0.98)^{1 / x}$. If we choose $x=0.588$ for good solvents, we get $H_{E} / H_{W}=1.30$. Our experimental result of $H_{E} / H_{W}$ was $1.24(=65.7 / 53.2)$.

The difference between 1.30 and 1.24 is about $5 \%$, therefore our theoretical calculation was consistent with our experimental results. Note that in our calculations, for pure solvents, we assume every repeat unit of PNiPAAm adsorbs a solvent molecule. This assumption implies that the brush thickness in pure ethanol $\left(V_{E}=1\right)$ is always thicker than in pure water $\left(V_{E}=0\right)$ due to the fact that molecular volume of ethanol is larger than water's.

\section{Appendix A.3. Definition of Chemical Potential Change}

Experimental chemical potential change used in this paper is defined as the same as in a standard Physical Chemistry book [43], namely:

$$
\mu=\ln \left[\frac{\gamma_{E} x_{E}}{\gamma_{w}\left(1-x_{E}\right)}\right]
$$

where $\gamma_{E}$ and $\gamma_{w}$ are activity coefficients for ethanol and water, respectively (they are functions of temperature and composition change), and $x_{E}$ is the molar fraction of ethanol in the ethanol-water mixture. The quantities $\gamma_{E}$ and $\gamma_{w}$ can be calculated by using the DDB Software Package [44].

\section{References and Note}

1. Zhang, Q.; Hoogenboom, R. Polymers with upper critical solution temperature behavior in alcohol/water solvent mixtures. Prog. Polym. Sci. 2015, 48, 122-142. [CrossRef]

2. Scherzinger, C.; Schwarz, A.; Bardowb, A.; Leonhardb, K.; Richtering, W. Cononsolvency of poly-N-isopropyl acrylamide (PNIPAM): Microgels versus linear chains and macrogels. Curr. Opin. Colloid Interface Sci. 2014, 19, 84-94. [CrossRef]

3. Winnik, F.M.; Ringsdorf, H.; Venzmer, J. Methanol-water as a co-nonsolvent system for poly(N-isopropylacrylamide). Macromolecules 1990, 23, 2415-2416. [CrossRef]

4. Amiya, T.; Hirokawa, Y.; Hirose, Y.; Li, Y.; Tanaka, T. Reentrant phase transition of N-isopropylacrylamide gels in mixed solvents. J. Chem. Phys. 1987, 86, 2375. [CrossRef]

5. Flory, P.J. Principles of Polymer Chemistry; Cornell University Press: Ithaca, NY, USA, 1953; ISBN 978-0-8014-0134-3.

6. Gennes, P.-G. Scaling Concepts in Polymer Physics; Cornell University Press: Ithaca, NY, USA, 1979; ISBN 978-0-8014-1203-5.

7. Zhulina, E.B.; Borisov, O.V.; Pryamitsyn, V.A.; Birshtein, T.M. Coil-globule type transitions in polymers. 1. Collapse of layers of grafted polymer chains. Macromolecules 1991, 24, 140-149. [CrossRef]

8. Zhulina, E.B.; Birshtein, T.M.; Borisov, O.V. Theory of ionizable polymer brushes. Macromolecules 1995, 28, $1491-1499$. [CrossRef]

9. Pryamitsyn, V.A.; Leermakers, F.A.M.; Fleer, G.J.; Zhulina, E.B. Theory of the collapse of the polyelectrolyte brush. Macromolecules 1996, 29, 8260-8270. [CrossRef]

10. Birshtein, T.M.; Amoskov, V.M.; Mercurieva, A.A.; Pryamitsyn, V.A. Phase transitions in polymer brushes. Macromol. Symp. 1997, 113, 151-161. [CrossRef]

11. Saphiannikova, M.G.; Pryamitsyn, V.A.; Cosgrove, T. Self-consistent brownian dynamics simulation of polymer brushes under shear. Macromolecules 1998, 31, 6662-6668. [CrossRef]

12. John, J.; Keating, I.V.; Joseph, I.; Georges, B. Polymer brushes for membrane separations: A review. ACS Appl. Mater. Interfaces 2016, 8, 28383-28399. 
13. Chen, W.; Cordero, R.; Tran, H.; Ober, C.K. 50th anniversary perspective: Polymer brushes: Novel surfaces for future materials. Macromolecules 2017, 50, 4089-4113. [CrossRef]

14. Nagase, K.; Okano, T.; Kanazawa, H. Poly(N-isopropylacrylamide) based thermoresponsive polymer brushes for bioseparation, cellular tissue fabrication, and nano actuators. Nano Struct. Nano Objects 2018, 16, 9-23. [CrossRef]

15. Liu, G.; Zhang, G. Reentrant behavior of poly(N-isopropylacrylamide) brushes in water-methanol mixtures investigated with a quartz crystal microbalance. Langmuir 2005, 21, 2086-2090. [CrossRef] [PubMed]

16. Edmondson, S.; Nguyen, N.T.; Lewis, A.L.; Armes, S.P. Co-nonsolvency effects for surface-initiated poly(2-(methacryloyloxy)ethyl phosphorylcholine) brushes in alcohol/water mixtures. Langmuir 2010, 26, 7216-7226. [CrossRef] [PubMed]

17. Sui, X.; Chen, Q.; Hempenius, M.A.; Vancso, G.J. Probing the collapse dynamics of poly(N-isopropylacrylamide) brushes by AFM: Effects of co-nonsolvency and grafting densities. Small 2011, 7, 1440-1447. [CrossRef] [PubMed]

18. Espinosa-Marzal, R.M.; Nalam, P.C.; Bolisetty, S.; Spencer, N.D. Impact of solvation on equilibrium conformation of polymer brushes in solvent mixtures. Soft Matter 2013, 9, 4045-4057. [CrossRef]

19. Yu, Y.; de la Cruz, R.A.L.; Kieviet, B.D.; Gojzewski, H.; Pons, A.C.; Vancso, J.; de Beer, S. Pick up, move and release of nanoparticles utilizing co-non-solvency of PNIPAM brushes. Nanoscale 2017, 9, 1670-1675. [CrossRef] [PubMed]

20. Chen, Q.; Kooij, E.S.; Sui, X.; Padberg, C.J.; Hempenius, M.A.; Schöna, P.M.; Vancso, G.J. Collapse from the top: Brushes of poly(N-isopropylacrylamide) in co-nonsolvent mixtures. Soft Matter 2014, 10, 3134-3142. [CrossRef] [PubMed]

21. Yu, Y.; Cirelli, M.; Kieviet, B.D.; Kooij, E.S.; Vancso, G.J.; de Beer, S. Tunable friction by employment of co-non-solvency of PNIPAM brushes. Polymer 2016, 102, 372-378. [CrossRef]

22. Yu, Y.; Kieviet, B.D.; Kutnyanszky, E.; Vancso, G.J.; de Beer, S. Cosolvency-induced switching of the adhesion between poly(methyl methacrylate) brushes. ACS Macro Lett. 2015, 4, 75-79. [CrossRef]

23. Nalam, P.C.; Daikhin, L.; Espinosa-Marzal, R.M.; Clasohm, J.; Urbakh, M.; Spencer, N.D. Two-fluid model for the interpretation of quartz crystal microbalance response: Tuning properties of polymer brushes with solvent mixtures. J. Phys. Chem. C 2013, 117, 4533-4543. [CrossRef]

24. Wang, H.; Pemberton, J.E. Effect of solvent quality on laminar slip flow penetration of poly(N-isopropylacrylamide) films with an exploration of the mass transport mechanism. Langmuir 2017, 33, 7468-7478. [CrossRef] [PubMed]

25. Mukherji, D.; Kremer, K. Coil-globule-coil transition of PNiPAm in aqueous methanol: Coupling all-atom simulations to semi-grand canonical coarse-grained reservoir. Macromolecules 2013, 46, 9158-9163. [CrossRef]

26. Tanaka, F.; Koga, T. Temperature-responsive polymers in mixed solvents: Competitive hydrogen bonds cause cononsolvency. Phys. Rev. Lett. 2008, 101, 028302. [CrossRef] [PubMed]

27. Mukherji, D.; Marques, C.M.; Kremer, K. Polymer collapse in miscible good solvents is a generic phenomenon driven by preferential adsorption. Nat. Commun. 2014, 5, 4882. [CrossRef] [PubMed]

28. Jens-Uwe, S. Adsorption-attraction model for co-nonsolvency in polymer brushes. Macromolecules 2017, 50, $2219-2228$.

29. Jens-Uwe, S. Gluonic and regulatory solvents: A paradigm for tunable phase segregation in polymers. Macromolecules 2018, 51, 3066-3074.

30. Chen, X.; Feng, W.; Han, X.; Liu, H. Possible way to study cononsolvency in confinement: A lattice density functional theory approach. Langmuir 2017, 33, 11446-11456. [CrossRef] [PubMed]

31. Rauch, S.; Eichhorn, K.; Oertel, U.; Stamm, M.; Kuckling, D.; Uhlmann, P. Temperature responsive polymer brushes with clicked rhodamine B: Synthesis, characterization and swelling dynamics studied by spectroscopic ellipsometry. Soft Matter 2012, 8, 10260-10270. [CrossRef]

32. Zdyrko, B.; Iyer, K.S.; Luzinov, I. Macromolecular anchoring layers for polymer grafting: Comparative study. Polymer 2006, 47, 272-279. [CrossRef]

33. Werner, A.C.; Eichhorn, K.J.; Grundke, K.; Simon, F.; Grahlert, W.; Jacobasch, H.J. Insights on structural variations of protein adsorption layers on hydrophobic fluorohydrocarbon polymers gained by spectroscopic ellipsometry (part I). Colloids Surfaces A Physicochem. Eng. Asp. 1999, 156, 3-17. [CrossRef]

34. Brittain, W.J.; Minko, S. A structural definition of polymer brushes. J. Polym. Sci. Part A Polym. Chem. 2007, 45, 3505-3512. [CrossRef]

35. Kubota, K.; Fujishige, S.; Ando, I. Solution properties of poly(N-isopropylacrylamide) in water. Polym. J. 1990, 22, 15-20. [CrossRef] 
36. Xue, C.; Yonet-Tanyeri, N.; Brouette, N.; Sferrazza, M.; Braun, P.V.; Leckband, D.E. Protein adsorption on poly(N-isopropylacrylamide) brushes: Dependence on grafting density and chain collapse. Langmuir 2011, 27, 8810-8818. [CrossRef] [PubMed]

37. Jentzsch, C.; Jens-Uwe, S. Polymer brushes in explicit poor solvents studied using a new variant of the bond fluctuation model. J. Chem. Phys. 2014, 141, 104908. [CrossRef] [PubMed]

38. Dalgicdir, C.; Rodríguez-Ropero, F.; van der Vegt, N.F.A. Computational calorimetry of PNIPAM cononsolvency in water/methanol mixtures. J. Phys. Chem. B 2017, 121, 7741-7748. [CrossRef] [PubMed]

39. Bentz, K.C.; Savin, D.A. Chain dispersity effects on brush properties of surface-grafted polycaprolactonemodified silica nanoparticles: Unique scaling behavior in the concentrated polymer brush regime. Macromolecules 2017, 50, 5565-5573. [CrossRef]

40. Qi, S.; Klushin, L.I.; Skvortsov, A.M.; Schmid, F. Polydisperse polymer brushes: Internal structure, critical behavior, and interaction with flow. Macromolecules 2016, 49, 9665-9683. [CrossRef]

41. De Vos, W.M.; Leermakers, F.A.M. Modeling the structure of a polydisperse polymer brush. Polymer 2009, 50, 305-316. [CrossRef]

42. The volume of a polymer's repeat unit can be estimated by using the formula of $v=M_{0} /\left(\rho N_{A}\right)$, where $M_{0}$ is the molecular weight of repeat unit of the polymer, $\rho$ is the polymer's melt density and $N_{A}$ is the Avogadro's number. Then the size of a repeat unit can be estimated as $d=2(3 v /(4 \pi))^{1 / 3}$.

43. Job, G.; Rüffler, R. Physical Chemistry from a Different Angle; Springer International Publishing: Cham, Switzerland, 2016; ISBN 978-3319156651.

44. DDB Software Package, DDBST GmbH. Available online: http://www.ddbst.de/software-package.html (accessed on 10 June 2018).

(C) 2018 by the authors. Licensee MDPI, Basel, Switzerland. This article is an open access article distributed under the terms and conditions of the Creative Commons Attribution (CC BY) license (http:/ / creativecommons.org/licenses/by/4.0/). 